\title{
THE DIMENSION OF CLOSED SETS IN THE STONE-ČECH COMPACTIFICATION
}

\author{
JAMES KEESLING
}

\begin{abstract}
In this paper properties of compacta $K$ in $\beta X \backslash X$ are studied for Lindelöf spaces $X$. If $\operatorname{dim} K=\infty$, then there is a mapping $f: K \rightarrow T^{c}$ such that $f$ is onto and every mapping homotopic to $f$ is onto. This implies that there is an essential family for $K$ consisting of $c$ disjoint pairs of closed sets. It also implies that if $K=\bigcup\left\{K_{\alpha} \mid \alpha<c\right\}$ with each $K_{\alpha}$ closed, then there is a $\beta$ such that $\operatorname{dim} K_{\beta}=\infty$.

Assume $K$ is a compactum in $\beta X \backslash X$ as above. Then if $\operatorname{dim} K=n$, there is a closed set $K^{\prime}$ in $K$ such that $\operatorname{dim} K^{\prime}=n$ and such that every nonempty $G_{\delta}$-set in $K^{\prime}$ contains an $n$-dimensional compactum. This holds for $n$ finite or infinite. If $\operatorname{dim} K=n$ and $K=\bigcup\left\{K_{\alpha} \mid \alpha<\omega_{1}\right\}$ with each $K_{\alpha}$ closed, then there must be a $\beta$ such that $\operatorname{dim} K_{\beta}=n$.
\end{abstract}

Introduction. This paper is a continuation of the work in [10-12] studying the topological properties of the Stone-Čech compactification. Here we consider closed subsets of $\beta X \backslash X$ and we are concerned with the dimension properties of these sets. In [11] it was shown that if $K$ is a compact subset of $\beta X \backslash X$ with $\operatorname{dim} K \geqslant n$, then there is a map $f: K \rightarrow T^{n}$, where $T^{n}$ is the $n$-dimensional torus, such that if $g$ is any map homotopic to $f$, then $g$ is onto $T^{n}$. This implies that the shape dimension of $K$ must be $S-\operatorname{dim} K \geqslant n$. Winslow used the results in [11] to show that for $n \geqslant 3$, there are $2^{c}$ nonhomeomorphic compacta in $\beta R^{n} \backslash R^{n}$ [17]. In this paper the techniques in [11] are refined and new techniques are developed to give further insight into the dimension of compacta in $\beta X \backslash X$, particularly infinite-dimensional ones. The following theorems are representative of the results of the paper.

THEOREM 1.1. Let $X$ be Lindelöf and $K \subset \beta X \backslash X$ be a compactum with $\operatorname{dim} K=\infty$. Then there is a map $f: K \rightarrow T^{c}$ such that $f$ is homotopically onto $T^{c}$. Here $T^{c}$ is the $c$-fold product of circles, where $c$ is the cardinality of the reals.

THEOREM 2.1. Let $X$ be Lindelöf and $K \subset \beta X \backslash X$ be a compactum with $\operatorname{dim} K=\infty$. Then there is a collection of $c$ disjoint pairs of closed sets $\left\{\left(C_{\alpha}, D_{\alpha}\right) \mid \alpha<c\right\}$ such that if $B_{\alpha}$ is a closed subset of $K$ separating $C_{\alpha}$ from $D_{\alpha}$ for each $\alpha$, then $\cap\left\{B_{\alpha} \mid \alpha<c\right\} \neq \varnothing$.

THEOREM 3.5. Let $X$ be Lindelöf and suppose that $K$ is an n-dimensional compact set contained in $\beta X \backslash X$ with $n$ finite. Then there is an $n$-dimensional set $K^{\prime}$ contained in

Received by the editors June 21, 1984 and, in revised form, January 7, 1986.

1980 Mathematics Subject Classification (1985 Revision). Primary 54D35, 54D40.

Key words and phrases. Stone-Čech compactification, Lindelöf space, dimension, homotopically onto, $c$-torus, essential family, sum theorem. 
$K$ such that if $L$ is any nonempty zero set in $K^{\prime}$, then $\operatorname{dim} L=n$. Also, if $G$ is any nonempty $G_{\delta}$ in $K^{\prime}$, then $G$ contains an n-dimensional compact set.

THEOREM 3.6. Let $X$ be Lindelöf with $K$ an infinite-dimensional compact subset of $\beta X \backslash X$. Then there is an infinite-dimensional closed set $K^{\prime} \subset K$ such that if $L$ is any zero set in $K^{\prime}$, then $\operatorname{dim} L=\infty$ and if $G$ is any nonempty $G_{\delta^{-}}$set in $K^{\prime}$, then $G$ contains an infinite-dimensional compactum.

THEOREM 4.1. Suppose that $\operatorname{dim} K=\infty$ where $K$ is a compact subset of $\beta X \backslash X$ with $X$ Lindelöf. Suppose that $K=\bigcup\left\{K_{\alpha} \mid \alpha<c\right\}$ with $K_{\alpha}$ closed for all $\alpha$. Then there is $a$ $\beta$ such that $\operatorname{dim} K_{\beta}=\infty$.

THEOREM 4.2. Suppose that $X$ is Lindelöf and that $K$ is a compact subset of $\beta X \backslash X$. Suppose that $\operatorname{dim} K=n$ and suppose that $K$ is a compact subset of $\beta X \backslash X$. Suppose that $\operatorname{dim} K=n$ and suppose that $K=\bigcup\left\{K_{\alpha} \mid \alpha<\omega_{1}\right\}$ with $K_{\alpha}$ closed for all $\alpha<\omega_{1}$. Then there is a $\beta$ such that $\operatorname{dim} K_{\beta}=n$.

The proofs of the last two theorems are quite different even though the statements are similar. Some other results and examples are also given in the paper.

Preliminaries. Let $X$ be a topological space. Then $X$ is said to be completely regular provided that for every $x \in X$ and closed set $F$ not containing $x$ there is a continuous function $f: X \rightarrow[0,1]$ with $f(x)=0$ and $f(y)=1$ for all $y \in F$. All spaces are assumed to be completely regular and Hausdorff in addition to any other assumptions expressly stated. Either Gillman and Jerison [4] or Walker [16] contain all that we assume about the Stone-Čech compactification $\beta X$ of a completely regular space $X$. A zero set in $X$ is a set $Z \subset X$ such that there is a continuous function $f: X \rightarrow[0,1]$ with $Z=f^{-1}(0)$. Zero sets play an important role in the theory of completely regular spaces and the Stone-Čech compactification.

This paper assumes almost no knowledge of shape theory. It is a continuation of [10-12] which use shape theory in the study of the Stone-Čech compactification. It continues the spirit of those papers in that it studies the Stone-Čech compactification by studying homotopy classes of maps into polyhedra and other nice spaces. The book by Mardešić and Segal [13] gives a good summary of shape theory for those interested.

In the realm of separable metric spaces Hurewicz and Wallman [6] is the preferred text for dimension theory. However, we are concerned with spaces that are far from metric. For such spaces Pears [15] is the best text for our purposes although Engelking [3], Isbell [7], and Nagata [14] are also useful. The Lebesgue covering dimension is denoted by $\operatorname{dim} X$.

We use lower case Greek letters to denote the ordinals, $\alpha, \beta, \gamma$, etc. The cardinals are denoted by $\omega_{0}, \omega_{1}, \omega_{2}, \ldots$ A cardinal is thought of as an initial ordinal, the first ordinal with the given cardinality. We also let $c=2^{\omega_{0}}$ denote the cardinality of the reals. By $T^{n}$ we mean the $n$-dimensional torus and by $T^{c}$ we mean the product $\Pi\left\{S^{1} \mid \alpha<c\right\}$. 
1. Maps onto $T^{c}$. In [11] it was shown that if $X$ is Lindelöf and $K$ is a compact subset of $\beta X \backslash X$ with $\operatorname{dim} K \geqslant n$, then there is a map $f: K \rightarrow T^{n}$ which is onto such that every map homotopic to $f$ is also onto. We say that $f$ is homotopically onto. If $\operatorname{dim} K=\infty$, this result can be adapted to show that there is a map $f: K \rightarrow T^{\omega_{0}}$ which is homotopically onto. In this section we prove a much stronger result. We show that there is a map $f: K \rightarrow T^{c}$ which is homotopically onto. We also improve the theorem for finite-dimensional $K$ (Theorem 1.3).

As noted earlier we think of $c=2^{\omega_{0}}$ as an initial ordinal. Let $A=\left\{\alpha_{1}, \ldots, \alpha_{n}\right\}$ be a finite set of ordinals all less than $c$ and let $f_{A}: K \rightarrow T^{n}$ be $f$ followed by the projection $\pi_{A}: T^{c} \rightarrow T^{n}$ onto the subproduct $\Pi\left\{S_{\alpha}^{1} \mid \alpha \in A\right\}$ of $T^{c}$.

1.1. THEOREM. Suppose that $X$ is Lindelöf and $K$ is a compactum contained in $\beta X \backslash X$ with $\operatorname{dim} K=\infty$. Then there is a map $f: K \rightarrow T^{c}$ such that for any finite number of factors $A=\left\{\alpha_{1}, \ldots, \alpha_{n}\right\}, f_{A}: K \rightarrow T^{n}$ is homotopically onto. The map $f$ must therefore be homotopically onto as well.

The last statement of the theorem follows from the next lemma.

1.2. LemMa. Let $X$ be a compact space and $Y$ be the inverse limit of the inverse system $\left\{Y_{\alpha}, \pi_{\alpha \beta}\right\}$. Then if $f_{\alpha}: X \rightarrow Y_{\alpha}$ is homotopically onto for all $\alpha$ and $\pi_{\alpha \beta} \circ f_{\beta}=f_{\alpha}$ for all $\beta>\alpha$, then the map $f: X \rightarrow Y$ induced by $\left\{f_{\alpha}\right\}$ will also be homotopically onto.

Proof. Suppose that $f$ is not homotopically onto. Then there is a homotopy of $f$ to a map $g: X \rightarrow Y$ such that $g$ is not onto. Let $g_{\alpha}: X \rightarrow Y_{\alpha}$ be $g$ followed by the projection $\pi_{\alpha}: Y \rightarrow Y_{\alpha}$. Then there must be a $\beta$ such that $g_{\beta}$ is not onto $Y_{\beta}$. However, since $f$ and $g$ are homotopic, so are $f_{\beta}$ and $g_{\beta}$. This contradicts the fact that $f_{\beta}$ was assumed to be homotopically onto.

Now $T^{c}$ is the inverse limit of all its finite subproducts. Thus the last statement of Theorem 1.1 follows from the first part. Next we state a finite-dimensional version of Theorem 1.1.

1.3. TheOREM. Suppose that $X$ is Lindelöf and $K \subset \beta X \backslash X$ is a compactum with $\operatorname{dim} K \geqslant n$. Then there is a map $f: K \rightarrow T^{c}$ such that for any finite number of factors $A=\left\{\alpha_{1}, \ldots, \alpha_{n}\right\}$ with $k \leqslant n, f_{A}: K \rightarrow T^{k}$ is homotopically onto.

We now state and prove some lemmas needed to prove Theorems 1.1 and 1.3. Lemma 1.4 is a special case of the well-known result that if $B$ is an uncountable subset of the reals, then $B$ has an algebraically independent subset $A$ of the same cardinality.

1.4. Lemma. Let $B$ be any subset of the reals of cardinality $c$. Then there is a set $A \subset B$ of cardinality $c$ such that if $n$ is any natural number and $C$ is any $n \times n$ matrix whose entries are $n^{2}$ distinct elements of $A$, then $\operatorname{det} C \neq 0$.

Proof. We use the maximal principle. Let $\mathscr{P}$ be the set of all subsets $A$ of $B$ such that if $n$ is any natural number and $C$ is any $n \times n$ matrix with $n^{2}$ distinct entries out of $A$, then $\operatorname{det} C \neq 0$. Clearly there is a maximal $A_{0} \in \mathscr{P}$. We claim that the cardinality of $A_{0}$ must be $c$. Suppose not. Then let $\mathscr{F}\left(A_{0}\right)$ be the subfield of the 
reals generated by $A_{0}$. The cardinality of $\mathscr{F}\left(A_{0}\right)$ is countably infinite if $A_{0}$ is finite or both $A_{0}$ and $\mathscr{F}\left(A_{0}\right)$ have the same cardinality. Thus $B \backslash \mathscr{F}\left(A_{0}\right)$ must have cardinality $c$. Let $x \in B \backslash \mathscr{F}\left(A_{0}\right)$.

Claim. The set $\{x\} \cup A_{0}$ has the property that $\operatorname{det} C \neq 0$ for any natural number $n$ and any $n \times n$ matrix $C$ with $n^{2}$ distinct elements from $\{x\} \cup A_{0}$.

Proof of Claim. Let $C$ be any such matrix. Let us denote the entries of $C$ by $a_{i j}$. Then

$$
\operatorname{det} C=\sum_{\pi} \operatorname{sign}(\pi) \cdot \prod_{i=1}^{n} a_{i \pi(i)},
$$

where $\pi$ runs through all permutations of $\{1, \ldots, n\}$. Consider the entries $a_{i j}$ in the matrix $C$. If no $a_{i j}$ equals $x$, then all of them are in $A_{0}$. But this would imply that $\operatorname{det} C \neq 0$. On the other hand, if $x$ is one of the entries, then there is exactly one element of $C$ equaling $x$. If $n=1$, then $C=(x)$ and $\operatorname{det} C=x \neq 0$ because $x \notin \mathscr{F}\left(A_{0}\right)$. Suppose that $n>1$ and that $a_{i_{0} j_{0}}=x$. In the formula for $\operatorname{det} C$, $x=a_{i_{0} j_{0}}$ appears in each of the product terms at most once. Thus $\operatorname{det} C=a x+b$ with $a$ and $b$ elements of $\mathscr{F}\left(A_{0}\right)$. If $a \neq 0$ and $\operatorname{det} C=0$, then $x=-b / a \in \mathscr{F}\left(A_{0}\right)$. This would contradict the fact that $x$ was chosen so that $x \notin \mathscr{F}\left(A_{0}\right)$. On the other hand $a=\sum_{\pi \in \mathscr{S}} \operatorname{sign}(\pi) \prod_{i \neq i_{0}} a_{i \pi(i)}$, where $\mathscr{S}=\{\pi$ a permutation of $\{1, \ldots, n\} \mid$ $\left.\pi\left(i_{0}\right)=j_{0}\right\}$. One can verify that $a=(-1)^{i_{0}+j_{0}} \operatorname{det} C^{\prime}$, where $C^{\prime}$ is the $(n-1) \times$ $(n-1)$ matrix obtained from $C$ by deleting the $i_{0}$ th row and the $j_{0}$ th column. Thus $a \neq 0$ since $C^{\prime}$ is an $(n-1) \times(n-1)$ matrix all of whose entries are from the set $A_{0}$. Thus det $C \neq 0$ in this case as well. Thus, whether $x$ is an entry in the matrix or not, $\operatorname{det} C \neq 0$. This proves the Claim.

The Claim is a contradiction of the maximality of $A_{0}$ since $\{x\} \cup A_{0}$ properly contains $A_{0}$.

For the next few lemmas let $B^{n}=\left\{x \in R^{n} \mid\|x\| \leqslant 1\right\}$.

1.5. Lemma. Let $X$ be a compact space. Then $\operatorname{dim} X \geqslant n$ if and only if there is an essential map $f: X \rightarrow B^{n}$.

This is a well-known characterization of dimension (see [7, p. 85] for instance). We will also need the following lemma concerning essential maps.

1.6. LemMA. Let $X$ be compact and suppose $f: X \rightarrow B^{n} \subset R^{n}$ is essential.

Then if $g: X \rightarrow R^{n}$ is such that $d(f, g)<\frac{1}{4}$, the image of $g$ contains all $x \in R^{n}$ with $\|x\| \leqslant \frac{1}{2}$.

Proof. Suppose $f$ is essential. Then if $A=f^{-1}\left(S^{n-1}\right) \subset X$, every extension of $f \mid A$ to all of $X$ is onto all of $B^{n}$ by the definition of an essential map. Let $r: R^{n} \rightarrow B^{n}$ be the radial retraction of $R^{n}$ onto $B^{n}$. Then $r \circ g: X \rightarrow B^{n}$ and $d(f, r \circ g)<\frac{1}{4}$ for any map $g: X \rightarrow R^{n}$ with $d(f, g)<\frac{1}{4}$. Let $H: X \times I \rightarrow B^{n}$ be 
the straight line homotopy between $f$ and $r \circ g, H(x, t)=t f(x)+(1-t) r$ $\circ g(x)$. Then let $h(x)$ be defined by

$$
h(x)= \begin{cases}(4\|f(x)\|-3) f(x)+(4-4\|f(x)\|) g(x), & \frac{3}{4} \leqslant\|f(x)\| \leqslant 1, \\ r \circ g(x), & \|f(x)\| \leqslant \frac{3}{4},\end{cases}
$$

Note that $h$ is continuous and that $h|A \equiv f| A$ since $\|f(x)\|=1$ for all $x \in A$. Note also that for $\mid h(x) \| \leqslant \frac{1}{2}, h(x)=r \circ g(x)=g(x)$. This is because if $\|h(x)\|$ $\leqslant \frac{1}{2}$, then $\|f(x)\| \leqslant \frac{3}{4}$. Now $h$ is an extension of $f \mid A$ to all of $X$. Thus $h$ must be onto $B^{n}$. In particular $h(X)$ must contain $\frac{1}{2} B^{n}=\left\{x \in B^{n} \mid\|x\| \leqslant \frac{1}{2}\right\}$. But this implies that $\frac{1}{2} B^{n}$ is in the image of $g$.

1.7. Lemma. Let $f$ and $g$ be two maps from $X$ to $T^{n}$. Then $\beta(f-g)=\beta f-\beta g$. Thus $\beta f$ and $\beta g$ are homotopic as maps from $\beta X$ to $T^{n}$ if and only if $\beta(f-g)$ is a null-homotopic map from $\beta X$ to $T^{n}$.

Proof. Consider $\beta f-\beta g$ (using the abelian topological group structure of $T^{n}$ ). Then $(\beta f-\beta g) \mid X \equiv f-g$. Thus $\beta(f-g) \equiv \beta f-\beta g$ since extensions from a dense subspace are unique. Clearly $\beta f$ is homotopic to $\beta g$ if and only if $\beta f-\beta g$ is null-homotopic.

1.8. LemMA. Let $X$ be any space and let $f$ and $g$ be two maps of $X$ onto $T^{n}$. Let $\varphi=f-g: X \rightarrow T^{n}$. Then $\beta f$ and $\beta g$ are homotopic if and only if $\varphi: X \rightarrow T^{n}$ has a lifting $\tilde{\varphi}$ to the covering space $R^{n}$ of $T^{n}$ such that the lifting is bounded.

Proof. Suppose that $e: R^{n} \rightarrow T^{n}$ is the covering map and suppose that $\varphi=f-g$ and that $\tilde{\varphi}$ is a lifting of $\varphi$ so that $f-g=e \circ \tilde{\varphi}$ with $\tilde{\varphi}$ bounded. Then consider $\beta \tilde{\varphi}: \beta X \rightarrow R^{n}$. This map exists because $\tilde{\varphi}$ is bounded. But $e \circ \beta \tilde{\varphi}$ is an extension of $\varphi=f-g: X \rightarrow T^{n}$ and thus $e \circ \beta \tilde{\varphi}=\beta(f-g)$. Since $R^{n}$ is contractible, $e \circ \beta \tilde{\varphi}$ is null-homotopic. Thus $\beta f-\beta g$ is null-homotopic and $\beta f$ and $\beta g$ are homotopic by Lemma 1.7.

Now suppose that $\beta f$ and $\beta g$ are homotopic. Then $\beta f-\beta g$ is null-homotopic. Thus $\beta(f-g)$ is null-homotopic and has a lifting to $R^{n}$. Let $\psi=\beta(f-g)$ and denote the lifting by $\tilde{\psi}$. Then $\tilde{\psi} \mid X=\tilde{\varphi}$ is a lifting of $\varphi=f-g$ to $R^{n}$. Clearly $\tilde{\varphi}$ is bounded since its image is contained in $\tilde{\psi}(\beta X)$ which is compact. This proves Lemma 1.8.

1.9. Proposition. Suppose that $A$ is any metric space which is an absolute extensor with respect to metric spaces. Then $A$ is also an absolute extensor with respect to the category of paracompact spaces.

Proof. Let $X$ be any paracompact space and $f: B \rightarrow A$ any mapping with $B$ any closed subset of $X$. Let $\left\{\mathscr{U}_{i}\right\}_{i=1}^{\infty}$ be a countable collection of open covers of $A$ which forms a basis for the uniformity on $A$ induced by the metric of $A$. We can assume that $\mathscr{U}_{i+1}<^{*} \mathscr{U}_{i}$ for all $i$. Let $\mathscr{V}_{i}^{\prime}=f^{-1}\left(\mathscr{U}_{i}\right)$ be the cover of the closed set $B$, which will be relatively open with respect to $B$, and let $V_{\alpha}$ be an open set in $X$ such that $V_{\alpha} \cap B=V_{\alpha}^{\prime}$ for each $V_{\alpha}^{\prime} \in \mathscr{V}_{i}^{\prime}$. Then let $\mathscr{V}_{i}=\mathscr{V}_{i}^{\prime} \cup\{X \backslash B\} . \mathscr{V}_{i}$ is then an open covering of $X$ for each $i$. A characterization of paracompactness is that each open cover has a star refinement. Using this fact, one can easily see that a collection of 
open covers of $X,\left\{\mathscr{W}_{i}\right\}_{i=1}^{\infty}$, exists with the following properties: (1) For each $i, \mathscr{W}_{i}$ is an open covering of $X$; (2) $\mathscr{W}_{i+1}<* \mathscr{W}_{i}$ for each $i$; and (3) $\mathscr{W}_{i}<* \mathscr{V}_{i}$ for each $i$. Now let $\approx$ be a relation defined on $X$ by $x \approx y$ if and only if $y \in \operatorname{star}\left(x, \mathscr{W}_{i}\right)$ for all $i$. One can easily see that $\approx$ is an equivalence relation on $X$. Let us denote the equivalence class of an element $x \in X$ by $[x]$. Now let $X^{\#}$ be the set of equivalence classes under the equivalence relation $\approx$ on $X$. For any $D \subset X$ let $D^{\#}=\{[x] \mid x \in$ $D\}$. For any collection of subsets $\mathscr{D}$ of $X$ let $\mathscr{D}^{\#}=\left\{D^{\#} \mid D \in \mathscr{D}\right\}$. Let $q$ be the function $q: X \rightarrow X^{\#}$ defined by $q(x)=[x]$. Then $q$ is uniformly continuous since one can easily see that $\mathscr{W}_{i}<q^{-1}\left(\mathscr{W}_{i}^{\#}\right)$ for each $i$. We now claim that $q^{-1}\left(\mathscr{W}_{i}^{\#}\right)<$ $\mathscr{W}_{i}^{*}$.

Claim 1. $q^{-1}\left(\mathscr{W}_{i}^{\#}\right)<\mathscr{W}_{i}^{*}$.

Proof of Claim 1. If $y \in q^{-1}\left(W^{\#}\right)$ for some $W^{\#} \in \mathscr{W}_{i}$, then $y \approx z$ for some $z \in W$. By the definition of $\approx$ we must have that $z \in \operatorname{star}\left(y, \mathscr{W}_{i}\right)$ and thus there is a $V \in \mathscr{W}_{i}$ such that $\{y, z\} \subset V$. Thus $y \in V$ and $z \in W \cap V$. Thus $y \in \operatorname{star}\left(W, \mathscr{W}_{i}\right)$ and $q^{-1}\left(\mathscr{W}_{i}^{\#}\right)<\mathscr{W}_{i}^{*}$ as asserted.

Now we claim that $\mathscr{W}_{i+2}^{\#}<* \mathscr{W}_{i}^{\#}$ and thus $\left\{\mathscr{W}_{i}^{\#}\right\}_{i=1}^{\infty}$ forms the basis for a metric uniformity on $X^{\#}$. We prove this now.

Claim 2. For all $i, \mathscr{W}_{i+2}^{\#}<* \mathscr{W}_{i}^{\#}$.

Proof of Claim 2. Suppose that $V^{\#}=\operatorname{star}\left(W_{0}^{\#}, \mathscr{W}_{i+2}^{\#}\right)$ for some $W_{0}^{\#} \in \mathscr{W}_{i+2}^{\#}$. Let $Q \in \mathscr{W}_{i}$ such that $\operatorname{star}\left(\operatorname{star}\left(W_{0}, \mathscr{W}_{i+2}\right), \mathscr{W}_{i+2}\right) \subset Q$. We will show that $V^{\#} \subset Q^{\#}$ and this will prove the claim. Let $[y] \in V^{\#}$. Then for some $W_{1}^{\#} \in \mathscr{W}_{i+2}^{\#},[y] \in W_{1}^{\#}$ with $W_{1}^{\#} \cap W_{0}^{\#} \neq \varnothing$. Let $w \approx y$ with $w \in W_{1}$. Now since $W_{1}^{\#} \cap W_{0}^{\#} \neq \varnothing$, we must have a $z \in W_{1}$ and a $v \in W_{0}$ such that $z \approx v$. Since $v \in \operatorname{star}\left(z, \mathscr{W}_{i+2}\right)$, there must be a $W_{2} \in \mathscr{W}_{i+2}$ such that $\{z, v\} \subset W_{2}$. This implies that $W_{0} \cap W_{2} \neq \varnothing$ and $W_{2} \cap W_{1} \neq \varnothing$. Thus $w \in W_{1} \subset \operatorname{star}\left(\operatorname{star}\left(W_{0}, \mathscr{W}_{i+2}\right), \mathscr{W}_{i+2}\right)$. But by the choice of $Q$ above we must have $w \in Q$. Since $y \approx w$ we must have that $[y] \in Q^{\#}$ and we have shown that $V^{\#} \subset Q^{\#}$ and Claim 2 is proved.

Let $B^{\#}=q(B)$. We now define a map $f^{\#}: B^{\#} \rightarrow A$ by $f^{\#}([x])=f(x)$ whenever $[x] \in B^{\#}$ and $x$ is a representative of this equivalence class which comes from $B$. We claim that $f^{\#}$ is well defined.

Claim 3. The function $f^{\#}: B^{\#} \rightarrow A$ is well defined.

Proof of Claim 3. Suppose that $f(x) \neq f(y)$ with $x, y \in B$. Then let $\mathscr{U}_{i}$ be such that $\operatorname{star}\left(f(x), \mathscr{U}_{i}\right) \cap \operatorname{star}\left(f(y), \mathscr{U}_{i}\right)=\varnothing$. For this $i, y$ is not an element of $\operatorname{star}\left(x, \mathscr{W}_{i}\right)$ since $\mathscr{W}_{i}<* \mathscr{V}_{i}$ and $\mathscr{V}_{i} \cap B=\mathscr{V}_{i}^{\prime}=f^{-1}\left(\mathscr{U}_{i}\right)$. Thus we cannot have $x \approx y$. Thus $x \approx y$ for $x$ and $y$ in $B$ implies that $f(x)=f(y)$ and Claim 3 is proved.

Now we claim that $\mathscr{W}_{i}^{\#} \cap B^{\#}<\mathscr{V}_{i}^{\#} \cap B^{\#}$.

Claim 4. $\mathscr{W}_{i}^{\#} \cap B^{\#}<\mathscr{V}_{i}^{\#} \cap B^{\#}$.

Proof of Claim 4. Let $W^{\#} \in \mathscr{W}_{i}^{\#}$. Then there is a $V \in \mathscr{V}_{i}$ such that $\operatorname{star}\left(W, \mathscr{W}_{i}\right) \subset V$ by property (3) of the covers $\left\{\mathscr{W}_{i}\right\}_{i=1}^{\infty}$. Now we claim that if $[y] \in W^{\#} \cap B^{\#}$, then $[y] \in V^{\#} \cap B^{\#}$. So, let $[y] \in W^{\#} \cap B^{\#}$. Then we may assume that $z \in W$ and $z \approx y \in B$. Now by the definition of $\approx, y \in \operatorname{star}\left(z, \mathscr{W}_{i}\right)$ and thus there must be a $W_{1} \in \mathscr{W}_{i}$ such that $\{y, z\} \subset W_{1}$. But this implies that $y \in \operatorname{star}\left(W, \mathscr{W}_{i}\right)$. Thus $y \in V$ and $[y] \in V^{\#}$. Thus $[y] \in V^{\#} \cap B^{\#}$ since $[y]$ is still in $B^{\#}$. We have now shown that $\mathscr{W}_{i}^{\#} \cap B^{\#}<\mathscr{V}_{i}^{\#} \cap B^{\#}$ and Claim 4 is proved. 
Claim 5. The map $f^{\#}$ is uniformly continuous.

Proof of Claim 5. Suppose that $\mathscr{U}_{i}$ is one of the covers in the basis of the uniformity on $A$. One can easily see that $f^{\#-1}\left(\mathscr{U}_{i}\right)=\left\{f^{-1}(U)^{\#} \mid U \in \mathscr{U}_{i}\right\}$. Thus $f^{\#-1}\left(\mathscr{U}_{i}\right)=f^{-1}\left(\mathscr{U}_{i}\right)^{\#}=\mathscr{V}_{i}^{\#} \cap B^{\#}$. But by Claim 4 we have $\mathscr{W}_{i}^{\#} \cap B^{\#}<\mathscr{V}_{i}^{\#} \cap$ $B^{\#}$. Thus $f^{\#}$ is uniformly continuous and Claim 5 is proved.

Now to complete the proof of Proposition 1.9, we note that since $f^{\#}$ is uniformly continuous it has an extension to the closure $\bar{B}^{\#}$ of $B^{\#}$. Call this extension $\bar{f}^{\#}$. But the mapping $\bar{f}^{\#}: \bar{B}^{\#} \rightarrow A$ is a continuous mapping from a closed subset of a metric space to the space $A$. The space $A$ was assumed to be an absolute extensor for metric spaces. Thus the mapping $\bar{f}^{\#}$ has an extension to the whole space $X^{\#}$. Call this extension $F^{\#}$. Now $F^{\#} \circ q\left|B=f^{\#} \circ q\right| B=f$. Thus if we let $F \equiv F^{\#} \circ q$, then $F$ will be a continuous extension for the map $f$ to the whole space $X$. Since $B$ and $f$ were arbitrary, we have shown that the space $A$ is an absolute extensor with respect to paracompact spaces.

1.10. Corollary. Suppose that $A$ is a metric space which is an absolute extensor with respect to metric spaces. Then $A$ is an absolute extensor with respect to (regular Hausdorff ) Lindelöf spaces as well.

Proof. Every regular Lindelöf space is paracompact and 1.10 follows from 1.9.

Proof of Theorem 1.1. Let $B_{i}=\left\{x_{i, \alpha} \mid \alpha<c\right\}$ for $i=1,2, \ldots$ be a countable collection of sets of $c$ real numbers in $(0,1)$ such that $x_{i, \alpha} \neq x_{j, \beta}$ for $(i, \alpha) \neq(j, \beta)$ and such that if $B=\bigcup\left\{B_{i} \mid i=1,2, \ldots\right\}$, then every $n \times n$ matrix $A$ formed by distinct elements of $B$ has $\operatorname{det} A \neq 0$. This collection exists by Lemma 1.4 and the fact that $\omega_{0} \cdot c=c$. Let $l_{1}=\left\{\left(r_{i}\right) \in R^{\omega_{0}}\left|\sum_{i=1}^{\infty}\right| r_{i} \mid<\infty\right\}$ be the Banach space of absolutely convergent sequences of real numbers. We now define a function $g: l_{1} \rightarrow$ $R^{c}=\prod\left\{R_{\alpha} \mid \alpha<c\right\}$ by $g\left(\left(r_{i}\right)\right)=\left(g_{\alpha}\left(\left(r_{i}\right)\right)\right)$, where $g_{\alpha}\left(\left(r_{i}\right)\right)=\sum_{i=1}^{\infty} r_{i} x_{i, \alpha}$.

Claim 1. The function $g: l_{1} \rightarrow R^{c}$ is continuous.

Proof of Claim 1. The function $g$ will be continuous provided that each $g_{\alpha}$ is continuous since $R^{c}$ is a product space. Now $g_{\alpha}: l_{1} \rightarrow R_{\alpha}$ is just a function between two metric spaces. Each $g_{\alpha}$ is linear and thus we need only check continuity at $0 \in l_{1}$. Let $\varepsilon>0$. Then let $\delta=\varepsilon$. Then for $\left(r_{i}\right) \in l_{1}$ with $d\left(\left(r_{i}\right), 0\right)<\delta, \sum_{i=1}^{\infty}\left|r_{i}\right|<\delta$ $=\varepsilon$. Thus

$$
\left|\sum_{i=1}^{\infty} r_{i} x_{i, \alpha}\right| \leqslant \sum_{i=1}^{\infty}\left|r_{i} x_{i, \alpha}\right| \leqslant \sum_{i=1}^{\infty}\left|r_{i}\right|<\varepsilon .
$$

Thus $g_{\alpha}$ is continuous for all $\alpha, g$ is continuous and Claim 1 is proved.

Continuing with the proof of Theorem 1.1 we let $l_{1, f}=\left\{\left(r_{i}\right) \in l_{1} \mid\right.$ for some $N$, $r_{i}=0$ for all $\left.i>N\right\}$. It is well known that $l_{1, f}$ is an absolute retract for metric spaces (see [5]). We will need this fact later.

Now let $K$ be an infinite-dimensional compactum in $\beta X \backslash X$ with $X$ Lindelöf.

Claim 2. There is a collection of compact connected sets $\left\{N_{i} \mid i=1,2, \ldots\right\}$ in $K$ and a set of open subsets $\left\{U_{i} \mid i=1,2, \ldots\right\}$ of $K$ with $N_{1} \subset U_{i}$ such that (1) $\operatorname{dim} N_{i} \geqslant i$ for all $i$ and (2) $U_{i} \cap N_{j}=\varnothing$ for all $i \neq j$. 
Proof of Claim 2. Suppose that there is an $x \in K$ such that for every neighborhood $U$ of $x$ in $K, \operatorname{dim} \bar{U}=\infty$. (We will later show that there must be such a point in $K$.) Then let $U$ be a neighborhood such that $\operatorname{dim}(K \backslash U) \geqslant 1$. Let $N_{1}$ be a component of $K \backslash U$ with $\operatorname{dim} N_{1} \geqslant 1$ and let $U_{1}$ be a neighborhood of $N_{1}$ in $K$ such that $x$ is not an element of $\bar{U}_{1}$. Then let $U$ be a neighborhood of $x$ such that $\left.\operatorname{dim}\left[\left(K \backslash U_{1}\right) \backslash U\right)\right] \geqslant 2$. Let $N_{2}$ be a component of $\left(K \backslash U_{1}\right) \backslash U$ with $\operatorname{dim} N_{2} \geqslant 2$ and let $U_{2}$ be a neighborhood of $N_{2}$ such that $\bar{U}_{2} \cap N_{1}=\varnothing$ with $x$ not in $\bar{U}_{2}$. We can continue this process inductively to get the desired collection.

Now we show that there must be an $x \in K$ such that for every neighborhood $U$ of $x$ in $K, \operatorname{dim} \bar{U}=\infty$. Suppose not. Then for every $x \in K$, there is a neighborhood $U_{x}$ of $x$ such that $\operatorname{dim} \bar{U}_{x}=n_{x}<\infty$. Now $K=\bigcup\left\{U_{x} \mid x \in K\right\}$. Since $K$ is compact, there is a finite subcover of this collection $\left\{U_{1}, \ldots, U_{n}\right\}$. But this implies that $\operatorname{dim} K \leqslant \max \left\{\operatorname{dim} \bar{U}_{i} \mid i=1,2, \ldots, n\right\}<\infty$ by the Sum Theorem for Lebesgue covering dimension for normal spaces. This contradiction implies that the required $x$ must exist and the proof of Claim 2 is complete.

Since the sets $N_{i}$ are contained in $K$, if we let $N=\bigcup\left\{N_{i} \mid i=1,2, \ldots\right\}$, then $\mathrm{Cl}_{\beta X} N \subset K$. Note that $N$ is $\sigma$-compact and thus $X \cup N$ is Lindelöf. Now $N$ is closed in $X \cup N$ (since $K \cap(X \cup N)=N$ and $K$ is compact). This implies that if $f: N \rightarrow Y$ is a map where $Y$ is an absolute extensor for Lindelöf spaces, then $f$ will have a continuous extension $\bar{f}: X \cup N \rightarrow Y$. We will be constructing a map $f: N \rightarrow$ $l_{1, f}$. We will want to extend the map to $\bar{f}: X \cup N \rightarrow l_{1, f}$. This will be possible by Corollary 1.10 since $l_{1, f}$ is an absolute extensor for metric spaces and thus for Lindelöf spaces as well. If we let $Y=[0,1]$ in the above, then if we have any $f: N \rightarrow[0,1]$, then we can find an $\bar{f}: X \cup N \rightarrow[0,1]$ extending $f$. But then we can extend $\bar{f}$ to $\beta \bar{f}: \beta X \rightarrow[0,1]$. But $\beta \bar{f} \mid \mathrm{Cl}_{\beta X} N$ is an extension of $f$ to $\mathrm{Cl}_{\beta X} N$. Since $f$ could be any map of $N$ into [0,1], this implies that $\mathrm{Cl}_{\beta X} N \approx \beta N$ by a well-known characterization of the Stone-Čech compactification.

Now we construct the map $p: N \rightarrow l_{1, f}$. Identify $R^{n}$ with the set $\left(r_{i}\right) \in l_{1}$ such that $r_{i}=0$ for all $i>n$. Then $R^{1} \subset R^{2} \subset \cdots \subset R^{n} \subset \cdots$ and $\bigcup\left\{R^{n} \mid n=\right.$ $1,2, \ldots\}=l_{1, f}$. For each $n$ there are an infinite number of disjoint sets, $N_{i}$, with $\operatorname{dim} N_{i} \geqslant n$. We break up the collection $\left\{N_{i} \mid i=1,2, \ldots\right\}$ into a countable collection of countably many sets $\left\{N_{i j} \mid i=1,2, \ldots ; j=1,2, \ldots\right\}$, where $\operatorname{dim} N_{i j} \geqslant i$ for all $j$. Now let $p_{i j}: N_{i j} \rightarrow B^{i}$ be an essential map. Such a map exists since $\operatorname{dim} N_{i j} \geqslant i$. Then let $N=\bigcup\left\{N_{i j} \mid i=1,2, \ldots ; j=1,2, \ldots\right\}$ and define $p: N \rightarrow l_{1, f}$ by $p \mid N_{i j} \equiv$ $j \cdot p_{i j}: N_{i j} \rightarrow j \cdot B^{i} \subset R^{i} \subset l_{1, f}$. Since $N_{i j}$ has a neighborhood $U_{i j}$ such that $U_{i j} \cap$ $N_{k m}=\varnothing$ for $(i, j) \neq(k, m)$, the map $p$ is well defined and continuous. By the above remarks we can extend $p$ to $\bar{p}: X \cup N \rightarrow i_{1, f}$. Now consider the map $g: l_{1, f} \rightarrow R^{c}$ which was shown to be continuous in Claim 1. Let $e: R \rightarrow S^{1}$ be the exponential map $e(t)=e^{2 \pi i t}$ and let $e^{c}: R^{c} \rightarrow T^{c}$ be the $c$-fold product of $e$. Then let $h: X \rightarrow T^{c}$ be $h=e^{c} \circ g \circ \bar{p} \mid X$.

$$
\begin{array}{cr}
X \stackrel{\bar{p} \mid X}{\rightarrow} & l_{1, f} \subset l_{1} \\
h \searrow & \\
& \downarrow g \\
& T^{c} \underset{e^{c}}{\leftarrow} R^{c}
\end{array}
$$


Since $T^{c}$ is compact, $h$ has an extension to $\beta X, \beta h: \beta X \rightarrow T^{c}$. Let $f=\beta h \mid K$. We will now show that $f$ is the map asserted to exist in Theorem 1.1.

Claim 3. Let $A=\left\{\alpha_{1}, \ldots, \alpha_{n}\right\}$ be any finite subset of $\{\alpha<c\}$ and let $f_{A}=$ $\pi_{A} \circ f: K \rightarrow T^{n}$. Then $f_{A}$ is homotopically onto.

Proof of Claim 3. Consider $f_{A}: K \rightarrow T^{n}$ and suppose that $b: K \rightarrow T^{n}$ is any map homotopic to $f_{A}$. Then $b-f_{A}$ is null-homotopic by Lemma 1.7. Now consider $b-f_{A} \mid \mathrm{Cl}_{\beta X} N$ and $b-f_{A} \mid N$. These maps must also be null-homotopic. By Lemma 1.8 there is a lifting of $b-f_{A} \mid N$ to $R^{n}$ such that the lift is bounded in $R^{n}$. Suppose the lift is bounded by $M$. This gives us a lifting of $b-f_{A} \mid N_{i j}$ for all $i$ and $j$. In particular we have a lifting of $b-f_{A} \mid N_{n k}$ for all $k$ where $n$ is the dimension of $T^{n}$. Now $f_{A} \mid N_{n k}$ also has a lifting, namely $\pi \circ g \circ p_{n k}$, as indicated by the following diagram where $\pi=\pi_{A}$.

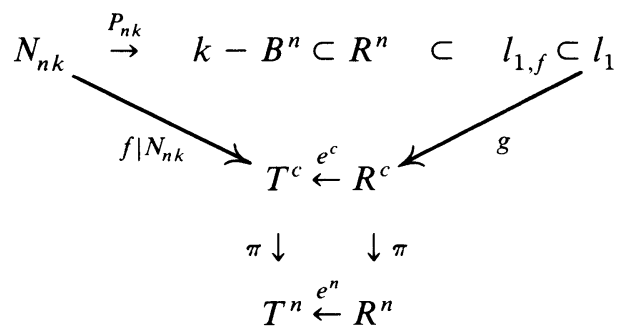

This implies that $\varphi=b \mid N_{n k}$ also has a lifting homotopic to $\pi \circ g \circ p_{n k}$. Call this lifting $\tilde{\varphi}$. Now $N_{n k}$ is connected and on connected spaces liftings are unique up to covering transformation. Thus the distance between $\pi \circ g \circ p_{n k}$ and $\tilde{\varphi}$ can be taken to be less than $M$. We need another claim before we can complete the proof of Claim 3.

Claim 4. The map $\pi \circ g \mid R^{n}: R^{n} \rightarrow R^{n}$ is onto.

Proof of Claim 4. Recall the definition of $g$. For $\left(r_{i}\right) \in l_{1}, g_{\alpha}\left(\left(r_{i}\right)\right)=\sum_{i=1}^{\infty} r_{i} x_{i, \alpha}$ and $g=\left(g_{\alpha}\right)_{\alpha<c}$. Now $\pi \circ g$ is just $\left(g_{\alpha}\right)_{\alpha \in A}$ and this restricted to $R^{n}$ is just a linear transformation from $R^{n}$ to $R^{n}$. Let $\left\{e_{j} \mid j=1, \ldots, n\right\}$ be the standard basis for $R^{n}$, $e_{j}=\left(\delta_{j 1}, \ldots, \delta_{j n}\right)$. Then, for $\lambda=\alpha_{i}, g_{\lambda}\left(e_{j}\right)=\sum_{i=1}^{\infty} \delta_{i j} x_{k, \lambda}=x_{j, \lambda}$. Thus the matrix representing this linear transformation is $G_{A}=\left(a_{i j}\right)$, where $a_{i j}=x_{j, \lambda}$ with $\lambda=\alpha_{i}$. But by our choices of the elements $x_{i, \alpha}$, we must have $\operatorname{det} G_{A} \neq 0$. Thus $\pi \circ g \mid R^{n}$ is an isomorphism of $R^{n}$ onto $R^{n}$. This proves Claim 4 .

We now resume the proof of Claim 3. Let $q: R^{n} \rightarrow R^{n}$ be the inverse of $\pi \circ g \mid R^{n}$. Then let $d=\|q\|$ be the operator norm of $q$. Then the distance between $p_{n k}$ and $q \circ \tilde{\varphi}$ is less than $d \cdot M$ since $q$ is uniformly continuous. Now let $k>\frac{1}{4} \cdot d \cdot M$. Then the map $1 / k \cdot p_{n k}$ is onto $1 / k \cdot k \cdot B^{n}=B^{n}$ and is essential. Also the map $1 / k \cdot q \circ \tilde{\varphi}$ is within $\frac{1}{4}$ of $1 / k \cdot p_{n k}$. Thus $1 / k \cdot q \circ \tilde{\varphi}$ is onto $\frac{1}{2} B^{n}$ by Lemma 1.6. Thus $q \circ \tilde{\varphi}$ is onto $k B^{n} / 2$. Since $k$ is arbitrary, $q \circ \tilde{\psi}$ is onto $R^{n}$ where $\psi=b \mid N$ and $\tilde{\psi}$ is the lifting of $b \mid N$ having the property that $\tilde{\psi} \mid N_{n k}=\tilde{\varphi}$. Thus $\tilde{\psi}$ is onto $R^{n}$ since $q$ is an isomorphism. This implies that $b \mid N$ is onto $T^{n}$. Thus $b(K)=T^{n}$ and $f$ is homotopically onto. This proves Claim 3 and completes the proof of Theorem 1.1. 
$\S \S 2$ and 4 of this paper contain applications of Theorem 1.1. In the remainder of this section we prove Theorem 1.3. The proof is similar enough to that of 1.1 that it is only sketched.

Proof of Theorem 1.3. Let $K$ be a compact subset of $\beta X \backslash X$ with $X$ Lindelöf. Suppose that $\operatorname{dim} K \geqslant n$ with $n$ finite. Let $\left\{N_{i} \mid i=1,2, \ldots\right\}$ be a collection of continua in $K$ and $\left\{U_{i} \mid i=1,2, \ldots\right\}$ open sets in $K$ such that (1) $\operatorname{dim} N_{i} \geqslant n$ for all $i$, (2) $N_{i} \subset U_{i}$ for all $i$, and (3) $U_{i} \cap N_{j}=\varnothing$ for all $i$ not equal to $j$. Let $N=\bigcup\left\{N_{i} \mid i=1,2, \ldots\right\}$. As in the proof of Theorem 1.1, any map $g: N \rightarrow R^{n}$ has an extension $\bar{g}: X \cup N \rightarrow R^{n}$ since $R^{n}$ is an absolute extensor. Let $B_{i}=\left\{x_{i, \alpha} \mid \alpha<\right.$ c) for $i=1,2, \ldots, n$ be sets of real numbers in $(0,1)$ such that for $B=\bigcup\left\{B_{i} \mid i=\right.$ $1,2, \ldots\}$, any $n \times n$ matrix $M$ made up of distinct elements of $B$ has $\operatorname{det} M \neq 0$. Let $g: R^{n} \rightarrow R^{c}$ be defined by $g=\left(g_{\alpha}\right)$, where $g_{\alpha}\left(r_{1}, \ldots, r_{n}\right)=r_{1} x_{1, \alpha}+\cdots+r_{n} x_{n, \alpha}$ $\in R_{\alpha}$. Then let $A=\left\{\alpha_{1}, \ldots, \alpha_{n}\right\}$ be any $n$ ordinals less than $c$ and let $\pi=\pi_{A}: R^{c}$ $\rightarrow R^{n}$ be the projection. Then $\pi \circ g$ is a linear transformation from $R^{n}$ to $R^{n}$. The matrix associated with this transformation is precisely $M=\left(a_{i j}\right)$, where $a_{i j}=x_{j, \lambda}$ with $\lambda=\alpha_{i}$. From the choice of the elements $x_{i, \alpha}$, det $M \neq 0$. Thus $\pi \circ g$ is an isomorphism for all $\pi=\pi_{A}$.

Now let $p_{i}: N_{i} \rightarrow B^{n}$ be an essential map, let $N=\bigcup\left\{N_{i} \mid i=1,2, \ldots\right\}$, and let $p: N \rightarrow R^{n}$ be defined by $p \mid N_{i}=i \cdot p_{i}$. Then $p$ will be well defined and continuous. Let $\bar{p}: X \cup N \rightarrow R^{n}$ be an extension of $p$ to $X \cup N$. Let $e^{c}: R^{c} \rightarrow T^{c}$ be the $c$-fold product of the exponential map $e: R \rightarrow S^{1}$. Then $e^{c} \circ g \circ \bar{p}$ is continuous and $T^{c}$ is a compact space. Thus there is an extension of $e^{c} \circ g \circ \bar{p}$ to $\beta X$ to $T^{c}$. Let $f: K \rightarrow T^{c}$ be the restriction of this map to $K$. Let $A=\left\{\alpha_{1}, \ldots, \alpha_{n}\right\}$ be any set of ordinals less than $c$ and let $\pi=\pi_{A}: R^{c} \rightarrow R^{n}$ be the projection. Let $\pi$ also denote the projection of $T^{c}$ onto $T^{n}$ for the same coordinates. Then in a fashion similar to the proof of Theorem 1.1, one can show that if $f_{A}=\pi \circ f: K \rightarrow T^{n}$ and $b$ is any map homotopic to $f_{A}$, then $b$ is onto all of $T^{n}$. Thus $f_{A}$ is homotopically onto. Now if $k \leqslant n$ it also follows that $f_{A}$ must be homotopically onto. This completes the proof.

2. Separating sets. In this section we show that if $K$ is a compactum in $\beta X \backslash X$ with $X$ Lindelöf, then $K$ is strongly infinite dimensional if $\operatorname{dim} K=\infty$. To be strongly infinite dimensional a space $X$ must have a countable collection of disjoint pairs of closed sets $\left\{\left(C_{i}, D_{i}\right) \mid i=1,2, \ldots\right\}$ in $X$ such that if $B_{i}$ is a closed set which separates $C_{i}$ and $D_{i}$ in $X$ for each $i$, then $\bigcap\left\{B_{i} \mid i=1,2, \ldots\right\} \neq \varnothing$. The Hilbert cube is strongly infinite dimensional with the pairs of disjoint closed sets being the pairs of opposite faces. Theorem 2.1 shows that $K$ has a property stronger than being strongly infinite dimensional.

2.1. TheOREM. Let $X$ be Lindelof and suppose that $K$ is a compact subset of $\beta X \backslash X$ with $\operatorname{dim} K=\infty$. Then there is a set of $c$ disjoint pairs of closed sets in $K$, $\left\{\left(C_{\alpha}, D_{\alpha}\right) \mid \alpha<c\right\}$, such that if $B_{\alpha}$ is a closed set in $K$ which separates $C_{\alpha}$ from $D_{\alpha}$ for all $\alpha<c$, then $\cap\left\{B_{\alpha} \mid \alpha<c\right\} \neq \varnothing$.

We will need a few propositions before we can prove the theorem. The first is a standard application of the Borsuk Extension Theorem and we omit the proof. 
2.2. Proposition. Let $X$ be any normal space such that $X \times I$ is normal. If $f: X \rightarrow I^{n}$ is essential, $g: X \rightarrow I^{n}$ is such that $g \mid f^{-1}\left(\partial I^{n}\right) \rightarrow \partial I^{n}$, and $g \mid f^{-1}\left(\partial I^{n}\right)$ is homotopic to $f \mid f^{-1}\left(\partial I^{n}\right)$ as maps into $\partial I^{n}$, then $g$ is essential.

2.3. Proposition. Let $X$ be any normal space. Suppose that $f: X \rightarrow I^{n}=[-1,1]^{n}$ is an essential mapping and let $C_{i}=f_{1}^{-1}(1)$ and $D_{i}=f_{i}^{-1}(-1)$. Then $\bigcap\left\{B_{i} \mid i=\right.$ $1,2, \ldots, n\} \neq \varnothing$ if $B_{i}$ is a closed set in $X$ which separates $C_{i}$ and $D_{i}$ for each $i=1,2, \ldots, n$.

Proof. Suppose that $B_{i}$ is a closed set in $X$ which separates $C_{i}$ and $D_{i}$ for $i=1,2, \ldots, n$. Suppose that $\bigcap\left\{B_{i} \mid i=1,2, \ldots\right\}=\varnothing$. Then we will first show that there are zero sets $Z_{i}$ such that $Z_{i}$ separates $C_{i}$ and $D_{i}$ with $B_{i} \subset Z_{i}$ and with $\bigcap\left\{Z_{i} \mid i=1,2, \ldots, n\right\}=\varnothing$. We prove this by induction on $n$. Suppose that $n=1$; then $B_{1}=\varnothing$ is a zero set. Proceed by induction and assume that whenever $\left\{B_{i} \mid i=1,2, \ldots, n\right\}$ are $n$ closed sets in a normal space with $\cap\left\{B_{i} \mid i=1,2, \ldots, n\right\}$ $=\varnothing$, then there are $n$ zero sets $Z_{i} \supset B_{i}$ for $i=1,2, \ldots, n$ such that $\cap\left\{Z_{i} \mid i=\right.$ $1,2, \ldots, n\}=\varnothing$. We will show that this is true for $n+1$ sets. Suppose that $\left\{B_{i} \mid i=1,2, \ldots, n+1\right\}$ is a collection of closed sets in a normal space with $\bigcap\left\{B_{i} \mid i=1,2, \ldots, n+1\right\}=\varnothing$. Then $B_{n+1}$ and $\bigcap\left\{B_{i} \mid i=1,2, \ldots, n\right\}$ are disjoint closed sets in a normal space. Let $Z_{n+1}$ be a zero set containing $B_{n+1}$ separating $C_{n+1}$ and $D_{n+1}$ and let $Z_{n}^{\prime}$ be a disjoint zero set with $Z_{n}^{\prime} \cap(C \cup D)=\varnothing$, where $C=\bigcup\left\{C_{i} \mid i=1,2, \ldots, n\right\}$ and $D=\bigcup\left\{D_{i} \mid i=1,2, \ldots, n\right\}$. We can also require that $Z_{n}^{\prime}$ contain a set $U$ which is the complement of a zero set in $X$ such that $U$ contains $\bigcap\left\{B_{i} \mid i=1,2, \ldots, n\right\}$. Then let $B_{i}^{\prime}=B_{i} \backslash U$ for $i=1,2, \ldots, n$. Then $\bigcap\left\{B_{i}^{\prime} \mid i=\right.$ $1,2, \ldots, n\}=\varnothing$ with $B_{i}^{\prime}$ separating $C_{i}$ from $D_{i}$ in $X \backslash U$. Let $Z_{i}^{\prime} \supset B_{i}^{\prime}$ be a zero set for $i=1,2, \ldots, n$ in $X \backslash U$ satisfying the induction hypothesis with $\cap\left\{Z_{i} \mid i=\right.$ $1,2, \ldots, n\}=\varnothing$. Then let $Z_{i}=Z_{i}^{\prime} \cup Z_{n}^{\prime}$ for $i=1,2, \ldots, n$. Then

$$
\bigcap\left\{Z_{i} \mid i=1,2, \ldots, n+1\right\}=Z \cap Z_{n+1}=Z^{\prime} \cap Z_{n+1}=\varnothing,
$$

where $Z=\bigcap\left\{Z_{i} \mid i=1,2, \ldots, n\right\}$ and $Z^{\prime}=\bigcap\left\{Z_{i}^{\prime} \mid i=1,2, \ldots, n\right\}$. Thus $\left\{Z_{i} \mid i=\right.$ $1,2, \ldots, n+1\}$ are the required zero sets. By induction we have shown that without loss of generality we may assume each $B_{i}$ to be a zero set separating $C_{i}$ from $D_{i}$ with $\bigcap\left\{B_{i} \mid i=1,2, \ldots, n\right\}=\varnothing$. Let $g_{i}: X \rightarrow[-1,1]$ be a continuous map with $g_{i}^{-1}(1) \supset$ $C_{i}$ and $g_{i}^{-1}(-1) \supset D_{i}$ with $g_{i}^{-1}(0)=B_{i}$. Then note that $g: X \rightarrow I^{n}$ defined by $g(x)=\left(g_{1}(x), \ldots, g_{n}(x)\right)$ has the property that $g(x) \neq(0, \ldots, 0)$ for all $x \in X$. But for $x \in C_{i}, g_{i}(x)=1$ and for $x \in D_{i}, g_{i}(x)=-1$. Thus $g \mid f^{-1}\left(\partial I^{n}\right)$ is homotopic to $f \mid f^{-1}\left(\partial I^{n}\right)$ as maps into $\partial I^{n}$. Since $g$ is not essential, $f$ must not be essential by Proposition 2.2. This is a contradiction. The contradiction implies that $\bigcap\left\{B_{i} \mid i=\right.$ $1,2, \ldots, n\} \neq \varnothing$ and Proposition 2.3 is proved.

2.4. Proposition. Let $X$ be a normal space and $f: X \rightarrow T^{n}$ be homotopically onto. Let $C_{i}=f_{i}^{-1}(1)$ and $D_{i}=f_{i}^{-1}(-1)$, where $f_{i}: X \rightarrow S^{1}$ is $f$ followed by projection onto the ith coordinate. Then if $B_{i}$ is a closed subset of $X$ separating $C_{i}$ from $D_{i}$ for each $i$, $\bigcap\left\{B_{i} \mid i=1,2, \ldots, n\right\} \neq \varnothing$. 
Proof. Suppose that $f: X \rightarrow T^{n}$ is as hypothesized and $C_{i}=f_{i}^{-1}(1)$ and $D_{i}=$ $f_{i}^{-1}(-1)$ for $i=1,2, \ldots, n$. Suppose that $B_{i}$ separates $C_{i}$ from $D_{i}$ for each $i$. Then let $E_{i}^{1}=f_{i}^{-1}\left(\left\{x \in S^{1} \mid \operatorname{Im}(x) \geqslant 0\right\}\right)$, let $E_{i}^{-1}=f_{i}^{-1}\left(\left\{x \in S^{1} \mid \operatorname{Im}(x) \leqslant 0\right\}\right)$, and let $(\delta(1), \ldots, \delta(n))$ be a sequence with each $\delta(i)$ equal to 1 or -1 . Let $A=A_{(\delta(1), \ldots, \delta(n))}$ $=\bigcap\left\{E_{i}^{\delta(i)} \mid i=1, \ldots, n\right\}$. Then $f(A)=I_{(\delta(1), \ldots, \delta(n))}^{n}=I^{n}$ is an $n$-cell in $T^{n}$ and $T^{n}$ is the union of $2^{n}$ such $n$-cells. Now consider $f \mid A: A \rightarrow I^{n}$. This map must be essential. Otherwise we could easily construct a map homotopic to $f$ which is not onto $T^{n}$. Now for each $(\delta(1), \ldots, \delta(n)), B_{i} \cap A$ separates $C_{i}$ from $D_{i}$ in $A$. If $A \cap\left(\cap\left\{B_{i} \mid i=1, \ldots, n\right\}\right)=\varnothing$, then $f \mid A$ would be inessential. Thus $A \cap$ $\left(\cap\left\{B_{i} \mid i=1, \ldots, n\right\}\right) \neq \varnothing$ for each $(\delta(1), \ldots, \delta(n))$.

ProOF OF THEOREM 2.1. For each $\alpha<c$, let $S_{\alpha}^{1}$ be the unit circle and $\pi_{\alpha}: T^{c} \rightarrow S_{\alpha}^{1}$ the coordinate projection. Then let $f: K \rightarrow T^{c}$ be the map which is given by Theorem 1.1 such that $f_{A}: K \rightarrow T^{n}$ is homotopically onto for every finite set of ordinals $A=\left\{\alpha_{1}, \ldots, \alpha_{n}\right\}$ with $\alpha_{i}<c$ for each $i$. Let $f_{\alpha}=\pi_{\alpha} \circ f, C_{\alpha}=f_{\alpha}^{-1}(1)$, and $D_{\alpha}=f_{\alpha}^{-1}(-1)$. Suppose that $B_{\alpha}$ separates $C_{\alpha}$ from $D_{\alpha}$ for each $\alpha$. Suppose that $\bigcap\left\{B_{\alpha} \mid \alpha<c\right\}=\varnothing$. Then there must be a finite collection $\left\{B_{\alpha(1)}, \ldots, B_{\alpha(n)}\right\}$ with $\bigcap\left\{B_{\alpha(i)} \mid i=1, \ldots, n\right\}=\varnothing$ by the compactness of $K$. But this contradicts Proposition 2.4 since $f_{A}$ is homotopically onto. Thus $\cap\left\{B_{\alpha} \mid \alpha<c\right\} \neq \varnothing$.

3. Zero sets. We recall the definition of zero set given in the preliminaries. Let $X$ be a space. A zero set in $X$ is a set $Z \subset X$ such that there is a continuous function $f: X \rightarrow[0,1]$ with $Z=f^{-1}(0)$. Zero sets form a basis for the closed sets in a completely regular space. In a normal space the zero sets are precisely the closed sets which are $G_{\delta}$ 's in the space. Thus in metric spaces all closed sets are zero sets. In any space if $\left\{L_{i} \mid i=1,2, \ldots\right\}$ is a countable collection of zero sets, then $\cap\left\{L_{i} \mid i=\right.$ $1,2, \ldots\}$ is also a zero set. In this section we present several theorems about the zero sets of closed sets in $\beta X \backslash X$.

3.1. THEOREM. Let $X$ be a compact space with $\operatorname{dim} X=n<\infty$. Then there is a closed set $K$ in $X$ with $\operatorname{dim} K=n$ with the property that if $L$ is any closed set in $K$ with nonempty interior in $K$, then $\operatorname{dim} L=n$.

To prove this we need the following standard result from dimension theory. It can be found in [15, p. 335, Lemma 1.7].

3.2. Lemma. Let $X$ be a compact space and A a closed subset of $X$. Let $f: X \rightarrow S^{n-1}$ be a continuous mapping which has no extension to $X$. Then there is a closed subset $K$ of $X$ such that $f$ has no extension to $A \cup K$, but $f$ has an extension to $A \cup L$ for every proper closed subset of $K$.

The next lemma is Proposition 2.3 in [15, p. 124].

3.3. Lemma. Let $A$ be a closed set of a normal space $X$ such that $\operatorname{dim} F \leqslant n$ for each closed $F$ of $X$ which is disjoint from $A$. Then each continuous $f: A \rightarrow S^{n}$ has an extension $\mathrm{g}: X \rightarrow S^{n}$.

Proof of Theorem 3.1. Since $X$ has dimension $n$, there is a map $f: A \rightarrow S^{n-1}$ with $A$ closed in $X$ such that there is no extension of $f$ to all of $X$. Let $K$ be the closed set given by Lemma 3.2, let $L$ be any closed subset of $K$ with nonempty 
interior, and suppose that $\operatorname{dim} L<n$. Let $U=$ int $_{K} L \neq \varnothing$. Then the map $f$ has an extension $\bar{f}$ to $A \cup(K \backslash U)$. Now any closed set $B$ in $K$ disjoint from $A \cup(K \backslash U)$ has dimension $\leqslant n-1$ since $B \subset U \subset L$. Thus $\bar{f}$ has an extension to $A \cup K$ by Lemma 3.3. This is a contradiction. Thus $\operatorname{dim} L=n$ and Theorem 3.1 is proved.

3.4. TheOREM. Let $n \geqslant 1$ be an integer. Let $X$ be a locally compact $\sigma$-compact space such that for every compact set $K \subset X$, there is a compact set $L \subset X \backslash K$ such that $\operatorname{dim} L \geqslant n$. Then $S-\operatorname{dim} \beta X \geqslant n$ and $S-\operatorname{dim}(\beta X \backslash X) \geqslant n$.

This is Theorem 1.6 of [11]. We note here that the shape dimension of a space, $S$-dim $X$, is always less than or equal to the Lebesgue dimension, $S$-dim $X \leqslant \operatorname{dim} X$. Thus Theorem 3.4 implies that $\operatorname{dim}(\beta X \backslash X) \geqslant n$.

3.5. THEOREM. Let $X$ be Lindelöf and suppose that $K$ is an $n$-dimensional compact set in $\beta X \backslash X$ with $n$ finite. Then there is an $n$-dimensional compact set $K^{\prime}$ in $K$ such that if $L$ is any nonempty zero set in $K^{\prime}$, then $G$ contains an $n$-dimensional compact set.

Proof. Suppose that $K$ is as above and let $K^{\prime}$ be the compact subset of $K$ having the property that if $L$ is closed in $K^{\prime}$ with nonempty interior, then $\operatorname{dim} L=n$. The set $K^{\prime}$ exists by Theorem 3.1. Let $L$ be a nonempty zero set in $K^{\prime}$. Let $f: K \rightarrow[0,1]$ be such that $f^{-1}(0)=L$. If $f^{-1}([0, \varepsilon])=L$ for some $\varepsilon>0$, then $L$ has nonempty interior in $K^{\prime}$ and thus $\operatorname{dim} L=n$. So, suppose that for all $\varepsilon>0$ there is a $y_{\varepsilon}$ not in $L$ with $f\left(y_{\varepsilon}\right) \in(0, \varepsilon)$. Let $y_{i}$ be a sequence such that each $y_{i}$ is not in $L$, but $f\left(y_{i}\right) \rightarrow 0$ with $f\left(y_{i}\right) \neq f\left(y_{j}\right)$ for all $i \neq j$. Let $I_{i}$ be a closed interval in $(0,1]$ containing $f\left(y_{i}\right)$ in its interior such that $I_{i} \cap I_{j}=\varnothing$ for all $i \neq j$. Let $L_{i}=f^{-1}\left(I_{i}\right)$. Then $L_{i}$ is a closed subset of $K^{\prime}$ and $y_{i} \in \operatorname{int}\left(L_{i}\right) \neq \varnothing$. Thus $\operatorname{dim} L_{i}=n$ for all $i$. Now $\bigcup\left\{L_{i} \mid i=1,2, \ldots\right\} \subset K^{\prime} \subset \beta X \backslash X$ with $X$ Lindelöf. Thus $\mathrm{Cl}_{K^{\prime}}\left(\bigcup\left\{L_{i} \mid i=1,2, \ldots\right\}\right)$ is homeomorphic to $\beta\left(\bigcup\left\{L_{i} \mid i=1,2, \ldots\right\}\right)$. Note also that $L_{i}$ is open and closed in $\bigcup\left\{L_{i} \mid i=1,2, \ldots\right\}$ and thus $\bigcup\left\{L_{i} \mid i=1,2, \ldots\right\}$ is locally compact and $\sigma$-compact. Thus $\bigcup\left\{L_{i} \mid i=1,2, \ldots\right\}$ satisfies the hypotheses of Theorem 3.4. Thus if $B=\mathrm{Cl}_{K^{\prime}}\left(\bigcup\left\{L_{i} \mid i=1,2, \ldots\right\}\right) \backslash \bigcup\left\{L_{i} \mid i=1,2, \ldots\right\}$, then $\operatorname{dim} B=n$. However, $f(B)=0$ since $f\left(L_{i}\right) \rightarrow 0$ and thus $B \subset f^{-1}(0)=L$. We have shown that $\operatorname{dim} L=n$.

Now suppose that $G$ is a nonempty $G_{\delta}$ in $K^{\prime}$. Let $G=\bigcap\left\{U_{i} \mid i=1,2, \ldots\right\}$ with each $U_{i}$ open in $K^{\prime}$ for all $i$ and let $x \in G$. For each $i$, there is a zero set $L_{i}$ such that $x \in L_{i} \subset U_{i}$. Then $x \in \bigcap\left\{L_{i} \mid i=1,2, \ldots\right\}=L$ is also a zero set with $L \subset G$. Since $L$ is nonempty, $\operatorname{dim} L=n$. This is the required $n$-dimensional compact set.

3.6. THEOREM. Let $X$ be Lindelöf and $K \subset \beta X \backslash X$ be any compact set with $\operatorname{dim} K=\infty$. Then there is a closed set $K^{\prime}$ in $K$ with the property that if $L$ is any zero set in $K^{\prime}$, then $\operatorname{dim} L=\infty$. Also, if $G$ is any nonempty $G_{\delta}$ set in $K^{\prime}$, then $G$ contains a compact set which is infinite dimensional.

Proof. Let $K$ be in $\beta X \backslash X$ as above. Let $\left\{L_{i} \mid i=1, \ldots\right\}$ be closed subsets of $K$ and $\left\{U_{i} \mid i=1, \ldots\right\}$ open subsets of $K$ such that (1) $\operatorname{dim} L_{i} \geqslant i$; (2) $U_{i} \supset L_{i}$ for all $i$; and (3) $U_{i} \cap L_{j}=\varnothing$ for all $i \neq j$. Such collections exist by the proof of Theorem 1.1. Now let $L_{i}^{\prime} \subset L_{i}$ be such that if $B \subset L_{i}^{\prime}$ is any closed set with nonempty interior 
in $L_{i}^{\prime}$, then $\operatorname{dim} B \geqslant i$. Such an $L_{i}^{\prime}$ exists by Theorem 3.3. Now let

$$
K^{\prime}=\mathrm{Cl}_{K}\left(\bigcup\left\{L_{i}^{\prime} \mid i=1, \ldots\right\}\right) \backslash \bigcup\left\{L_{i}^{\prime} \mid i=1, \ldots\right\} .
$$

Then by Theorem $3.4, \operatorname{dim} K^{\prime} \geqslant n$ for all $n$. Thus $\operatorname{dim} K^{\prime}=\infty$. We will now show that $K^{\prime}$ has the required properties. Let $L \subset K^{\prime}$ be any zero set. Let $f: K^{\prime} \rightarrow[0,1]$ be such that $f^{-1}(0)=L$. Now we can extend $f$ to

$$
\bar{f}: \mathrm{Cl}_{\beta X}\left(\cup\left\{L_{i} \mid i=1, \ldots\right\}\right) \rightarrow[0,1]
$$

with $\bar{f}\left(L_{i}^{\prime}\right) \subset(1 / i, 1]$ for all $i$. Now let $x_{n} \in L_{i(n)}^{\prime}$ be such that $\bar{f}\left(x_{n}\right) \rightarrow 0$ with all $\bar{f}\left(x_{n}\right)$ 's distinct. Let $I_{n}$ be a closed interval in $(0,1]$ containing $\bar{f}\left(x_{n}\right)$ in its interior such that $I_{n} \cap I_{m}=\varnothing$ for $n \neq m$. Then $\bar{f}^{-1}\left(I_{n}\right) \cap L_{i(n)}^{\prime}$ has nonempty interior in $L_{i(n)}^{\prime}$. Thus $\operatorname{dim} \bar{f}^{-1}\left(I_{n}\right) \cap L_{i(n)}^{\prime} \geqslant n$, so $\operatorname{dim} \bar{f}^{-1}\left(I_{n}\right) \geqslant n$ for all $n$. Let $B_{n}=\bar{f}^{-1}\left(I_{n}\right)$. Let $B=\mathrm{Cl}_{\beta X}\left(\bigcup\left\{B_{i} \mid i=1, \ldots\right\}\right) \backslash \bigcup\left\{B_{i} \mid i=1, \ldots\right\}$. Then $\operatorname{dim} B \geqslant n$ for all $n$ and thus $\operatorname{dim} B=\infty$. However, $\bar{f} \mid B \equiv 0$. Thus $B \subset L$ and $\operatorname{dim} L=\infty$. This proves that every nonempty zero set $L$ in $K^{\prime}$ has $\operatorname{dim} L=\infty$. Now suppose that $G$ is any $G_{\delta}$-set in $K^{\prime}$ which is nonempty. Then as in the proof of Theorem 3.5, there is a nonempty zero set $L$ contained in $G$. Then $L$ is the compact set with $\operatorname{dim} L=\infty$.

3.7. EXAMPLE. Let $K_{n}=\beta R^{n} \backslash R^{n}$. The proof of Theorem 3.5 implies that every nonempty zero set $L$ in $K_{n}$ has $\operatorname{dim} L=n$. Let $R^{k}$ be a $k$-dimensional linear subspace of $R^{n}$ with $k<n$. Then $\mathrm{Cl}_{\beta X} R^{k}$ is homeomorphic to $\beta R^{k}$. Thus $\beta R^{k} \backslash R^{k}$ $=K_{k}$ embeds as a closed subset of $K_{n}$. Although every nonempty zero set in $K_{n}$ has dimension $n$, there are $k$-dimensional closed subsets for every $k<n$.

4. Sum Theorems. Let $X$ be any normal space. The classical Sum Theorem for dimension theory is that if $X=\bigcup\left\{A_{i} \mid i=1,2, \ldots\right\}$ with each $A_{i}$ closed and $\operatorname{dim} A_{i} \leqslant n$ for each $i$, then $\operatorname{dim} X \leqslant n$. In this section we show that there is a stronger theorem for compact sets in $\beta X \backslash X$. There is a better theorem for $n$ infinite (Theorem 4.1) than for $n$ finite (Theorem 4.2) and the proofs in these two cases are quite different.

4.1. TheOREM. Suppose that $X$ is Lindelöf and that $K$ is a compactum in $\beta X \backslash X$. Suppose that $\operatorname{dim} K=\infty$ and that $K=\bigcup\left\{K_{\alpha} \mid \alpha<c\right\}$ with each $K_{\alpha}$ closed in $K$. Then there is a $\beta$ such that $\operatorname{dim} K_{\beta}=\infty$.

Proof. Suppose not. Then let $K=\bigcup\left\{K_{\alpha} \mid \alpha<c\right\}$ such that for all $\alpha, \operatorname{dim} K_{\alpha}<\infty$. Let $\left\{\left(C_{\alpha}, D_{\alpha}\right) \mid \alpha<c\right\}$ be the set of $c$ disjoint pairs of closed sets in $K$ given by Theorem 2.1. Let $\mathscr{A}$ be the set of all finite subsets of $\{\alpha<c\}$ and let $\left\{S_{\alpha} \mid \alpha<c\right\}$ be a collection of disjoint finite sets in $\{\alpha<c\}$ with card $S_{\alpha}>\operatorname{dim} K_{\alpha}$ for all $\alpha$ and with $\bigcup\left\{S_{\alpha} \mid \alpha<c\right\}=\{\alpha<c\}$. Then let $f:\{\alpha<c\} \rightarrow \mathscr{A}$ be defined by $f(\alpha)=S_{\alpha}$. Now for each $\alpha<c$ let $g_{\alpha}: K \rightarrow[-1,1]$ be a continuous function such that $g_{\alpha}^{-1}(-1)$ $\supset C_{\alpha}$ and $g_{\alpha}^{-1}(1) \supset D_{\alpha}$. Now let $\alpha<c$ and $n(\alpha)=\operatorname{dim} K_{\alpha}$ and let $F_{\alpha}: K \rightarrow$ $\Pi\{[-1,1] \lambda \in f(\alpha)\}=[-1,1]^{n(\alpha)}$ be defined by $F_{\alpha}(x)=\left(g_{\lambda}(x)\right)_{\lambda \in f(\alpha)}$. Now $F_{\alpha} \mid K_{\alpha}$ is not essential since $\operatorname{dim} K_{\alpha}<\operatorname{card} f(\alpha)$. Thus there is a map $G_{\alpha}: K_{\alpha} \rightarrow \partial\left([-1,1]^{n(\alpha)}\right)$ such that

$$
G_{\alpha}\left|F_{\alpha}^{-1}\left(\partial\left([-1,1]^{n(\alpha)}\right)\right) \equiv F_{\alpha}\right| F_{\alpha}^{-1}\left(\partial\left([-1,1]^{n(\alpha)}\right)\right) .
$$


Let $K_{\alpha}^{\prime}=K_{\alpha} \cup \mathscr{C}_{\alpha} \cup \mathscr{D}_{\alpha}$, where $\mathscr{C}_{\alpha}=\bigcup\left\{C_{\lambda} \mid \lambda \in f(\alpha)\right\} \quad$ and $\quad \mathscr{D}_{\alpha}=\bigcup\left\{D_{\lambda} \mid \lambda \in\right.$ $f(\alpha)\}$. Let $H_{\alpha}: K_{\alpha}^{\prime} \rightarrow[-1,1]^{n(\alpha)}$ be defined by

$$
H_{\alpha}(x)= \begin{cases}F_{\alpha}(x), & x \in \mathscr{C}_{\alpha} \cup \mathscr{D}_{\alpha}, \\ G_{\alpha}(x), & x \in K_{\alpha} .\end{cases}
$$

Then $H_{\alpha}$ is easily seen to be well defined and continuous. Let $H_{\alpha}^{\prime}$ be any extension of $H_{\alpha}$ to all of $K, H_{\alpha}^{\prime}: K \rightarrow[-1,1]^{n(\alpha)}$. Now for each $\lambda \in f(\alpha)$, let $h_{\lambda}: K \rightarrow[-1,1]$ be defined by $\pi_{\lambda} \circ H_{\alpha}^{\prime}: K \rightarrow[-1,1]$. Then for each $\lambda \in f(\alpha), h_{\lambda}^{-1}(-1) \supset C_{\lambda}$ and $h_{\lambda}^{-1}(1) \supset D_{\lambda}$. If we define $B_{\lambda}=h_{\lambda}^{-1}(0)$, then $B_{\lambda}$ will be a closed set in $K$ which separates $C_{\lambda}$ and $D_{\lambda}$ in $K$. We also claim that $K_{\alpha} \cap\left(\cap\left\{B_{\lambda} \mid \lambda \in f(\alpha)\right\}=\varnothing\right.$.

Claim. $K_{\alpha} \cap\left(\cap\left\{B_{\lambda} \mid \lambda \in f(\alpha)\right\}=\varnothing\right.$.

Proof of Claim. Suppose that $x \in K_{\alpha}$ and that $x \in \bigcap\left\{B_{\lambda} \mid \lambda \in f(\alpha)\right\}$. Then $H_{\alpha}^{\prime}(x)=H_{\alpha}(x)=G_{\alpha}(x)$ since $x \in K_{\alpha}$. Thus $G_{\alpha}(x) \in \partial\left([-1,1]^{n(\alpha)}\right)$ and $G_{\alpha}(x) \neq$ $(0,0, \ldots, 0)$. This implies that $x \notin \bigcap\left\{B_{\lambda} \mid \lambda \in f(\alpha)\right\}$ since $B_{\lambda}=h_{\lambda}^{-1}(0)$ for each $\lambda \in f(\alpha)$.

We have now shown that there exists a family of closed subsets of $K,\left\{B_{\alpha} \mid \alpha<c\right\}$, such that (1) $B_{\alpha}$ separates $C_{\alpha}$ and $D_{\alpha}$ in $K$ for all $\alpha<c$ and (2) $\left(\cap\left\{B_{\lambda} \mid \lambda \in f(\alpha)\right\}\right)$ $\cap K_{\alpha}=\varnothing$ for all $\alpha<c$. Now note that $\cap\left\{B_{\alpha} \mid \alpha<c\right\} \neq \varnothing$ by Theorem 2.1. However, $K_{\beta} \cap\left(\cap\left\{B_{\alpha} \mid \alpha<c\right\}\right)=\varnothing$ for each $\beta<c$ by (2). This implies that $K$ is not the union of the $K_{\alpha}$ 's, a contradiction. This implies that there must be a $\beta$ such that $\operatorname{dim} K_{\beta}=\infty$.

Now to prove the finite-dimensional version of Theorem 4.1, we will use the results of $\S 3$.

4.2. Theorem. Suppose that $X$ is Lindelof and that $K$ is a compact subset of $\beta X \backslash X$. Suppose that $\operatorname{dim} K=n$ and suppose that $K=\bigcup\left\{K_{\alpha} \mid \alpha<\omega_{1}\right\}$ with $K_{\alpha}$ closed for all $\alpha<\omega_{1}$. Then there is $a \beta$ with $\operatorname{dim} K_{\beta}=n$.

Proof. Let $K^{\prime} \subset K$ be an $n$-dimensional closed subset of $K$ with the property that every nonempty zero set $L$ in $K^{\prime}$ has $\operatorname{dim} L=n$. Without loss of generality we may assume $K^{\prime}=K$. Assume that $K=\bigcup\left\{K_{\alpha} \mid \alpha<\omega_{1}\right\}$ with each $K_{\alpha}$ closed and with $\operatorname{dim} K_{\alpha}<n$. Let $x \in K \mid K_{0}$. This point exists since $\operatorname{dim} K_{0}<n$. Let $f: K \rightarrow$ $[0,1]$ be such that $f(x)=0$ and $f\left(K_{0}\right)=1$. Then let $L_{0}=f^{-1}(0)$. Then $\operatorname{dim} L_{0}=n$ since $L_{0}$ is a nonempty zero set in $K$. Now suppose that we have $\left\{L_{\alpha} \mid \alpha<\beta\right\}$ defined for some $\beta<\omega_{1}$ such that (1) $L_{\alpha}$ is a nonempty zero set for each $\alpha$; (2) $L_{\gamma} \subset L_{\alpha}$ for $\alpha<\gamma<\beta$; and (3) $L_{\alpha} \cap K_{\alpha}=\varnothing$ for each $\alpha<\beta$. Then let $L=$ $\bigcap\left\{L_{\alpha} \mid \alpha<\beta\right\}$. Then $L$ is a zero set since $\{\alpha<\beta\}$ is countable. Also $L \neq \varnothing$, since $\left\{L_{\alpha} \mid \alpha<\beta\right\}$ has the finite intersection property and $K$ is compact. Thus $\operatorname{dim} L=n$. Thus $L \backslash K_{\beta} \neq \varnothing$ since $\operatorname{dim} K_{\beta}<n$. Let $x \in L \backslash K_{\beta}$ and let $f: K \rightarrow[0,1]$ be such that $f(x)=0$ and $f \mid K_{\beta} \equiv 1$. Then let $L_{\beta}=f^{-1}(0) \cap L$. Then $\left\{L_{\alpha} \mid \alpha<\beta+1\right\}$ satisfies (1), (2), and (3). We can continue this process for all $\alpha<\omega_{1}$ to get $\left\{L_{\alpha} \mid \alpha<\omega_{1}\right\}$ having properties (1), (2), and (3). But $\left\{L_{\alpha} \mid \alpha<\omega_{1}\right\}$ has the finite intersection property. Thus $\bigcap\left\{L_{\alpha} \mid \alpha<\omega_{1}\right\} \neq \varnothing$. However, $x \in \cap\left\{L_{\alpha} \mid \alpha<\omega_{1}\right\}$ implies that $x$ is not an element of $K_{\alpha}$ for each $\alpha$ since $L_{\alpha} \cap K_{\alpha}=\varnothing$ for each $\alpha$. Thus $K$ cannot be the union of the $K_{\alpha}$ 's. This contradiction implies that $\operatorname{dim} K_{\beta}=n$ for some $\beta<\omega_{1}$. 


\section{REFERENCES}

1. A. Calder and J. Siegel, Homotopy and uniform homotopy, Trans. Amer. Math. Soc. 235 (1978), 245-269.

2. __ Homotopy and uniform homotopy. II, Proc. Amer. Math. Soc. 78 (1980), 288-290.

3. R. Engelking, Dimension theory, North-Holland, Amsterdam, 1978.

4. L. Gillman and M. Jerison, Rings of continuous functions, Van Nostrand, Princeton, N. J., 1960.

5. W. E. Haver, Locally contractible spaces that are absolute neighborhood retracts, Proc. Amer. Math. Soc. 40 (1978), 280-284.

6. W. Hurewicz and H. Wallman, Dimension theory, Princeton Univ. Press, Princeton, N. J., 1941.

7. J. R. Isbell, Uniform spaces, Amer. Math. Soc., Providence, R. I., 1964.

8. J. Keesling, Shape theory and compact connected abelian topological groups, Trans. Amer. Math. Soc. 194 (1974), 349-358.

9. __ Continuous functions induced by shape morphisms, Proc. Amer. Math. Soc. 41 (1973), 315-320.

10. Decompositions of the Stone-Čech compactification which are shape equivalences, Pacific J. Math. 75 (1978), 455-466.

11. The Stone-Čech compactification and shape dimension, Topology Proc. 2 (1977), 483-508.

12. J. Keesling and R. B. Sher, Shape properties of the Stone-Čech compactification, General Topology App. 9 (1978), 1-8.

13. S. Mardešić and J. Segal, Shape theory, North-Holland, Amsterdam, 1982.

14. J. Nagata, Modern dimension theory, Wiley, New York, 1965.

15. A. R. Pears, Dimension theory of general spaces, Cambridge Univ. Press, Cambridge, 1975.

16. R. C. Walker, The Stone-Čech compactification, Springer-Verlag, New York, 1974.

17. A. Winslow, There are $2^{c}$ nonhomeomorphic continua in $\beta R^{n} \backslash R^{n}$, Pacific J. Math. 84 (1979), 233-239.

Department of Mathematics, University of Florida, Gainesville, Florida 32611 